\title{
BOUNDED SOLUTIONS OF CARATHÉODORY DIFFERENTIAL INCLUSIONS: A BOUND SETS APPROACH
}

JAN ANDRES, LUISA MALAGUTI, AND VALENTINA TADDEI

Received 9 March 2002

A bound sets technique is developed for Floquet problems of Carathéodory differential inclusions. It relies on the construction of either continuous or locally Lipschitzian Lyapunov-like bounding functions. Proceeding sequentially, the existence of bounded trajectories is then obtained. Nontrivial examples are supplied to illustrate our approach.

\section{Introduction}

This paper is mainly devoted to the investigation of bounded solutions of the differential inclusion

$$
x^{\prime} \in F(t, x), \quad t \in \mathbb{R} .
$$

We will always consider a Carathéodory set-valued map $F: \mathbb{R} \times \mathbb{R}^{N} \rightsquigarrow \mathbb{R}^{N}$ with nonempty, compact, and convex values. We recall that $F$ is said to be a Carathéodory multifunction if $F(\cdot, x)$ is measurable for each $x \in \mathbb{R}^{N}$, and $F(t, \cdot)$ is upper semicontinuous for almost all (a.a.) $t \in \mathbb{R}$. For the definitions of these standard notions, see, for example, [30].

Our main result is Theorem 4.2. It states the existence of a bounded solution for (1.1). The technique employed in its proof is essentially split into two parts.

First, in Section 3 (see Theorem 3.2), given an arbitrary real interval $[a, b]$, we solve the Floquet boundary value problem

$$
\begin{gathered}
x^{\prime} \in F(t, x), \quad \text { for a.a. } t \in[a, b], \\
x(b)=M x(a),
\end{gathered}
$$

where $M$ is a regular $N \times N$ matrix. We obtain its solvability by means of a continuation principle due to Andres et al. [7, Proposition 3.1] (see also [5, 8]). 
Such an approach requires, in particular, the existence of a bounded retract $Q$ of the Banach space $C\left([a, b], \mathbb{R}^{N}\right)$ satisfying suitable transversality conditions on its boundary. Thanks to the bound sets technique used in order to guarantee those conditions, we get a solution of (1.2) belonging to a given bounded subset of $C\left([a, b], \mathbb{R}^{N}\right)$.

Then, in Section 4 , we take $[a, b]=[-n, n]$ for each $n \in \mathbb{N}$, and by means of a classical sequential approach (the diagonalization argument), we get a solution of (1.1) which is bounded on the whole real line.

If the matrix $M$ is the identity, then (1.2) essentially reduces to the study of periodic solutions. This problem is well known and has been recently investigated by several authors with different techniques.

We quote, in particular, the results by Macki et al. [37]; they used a topological degree argument in order to obtain a periodic solution for the Carathéodory system $x^{\prime}+A(t) x \in F(t, x)$, under suitable growth conditions at zero and infinity.

Plaskacz [46] investigated (1.1) in the case of a globally measurable inclusion $F$, upper semicontinuous on its state variable $x$; the technique employed was again topological, involving the generalized Lefschetz fixed-point theorem.

In Górniewicz and Plaskacz [28], a Carathéodory inclusion is considered and the existence of a periodic solution follows also by employing a $C^{1}$-guiding function.

Castaing and Monteiro Marques [17, 18] obtained absolutely continuous and BV periodic solutions for single- and multivalued perturbations of the sweeping process of J. J. Moreau.

The bound sets theory was initiated by Gaines and Mawhin [27] (see also [41]) with smooth Lyapunov-like functions, called bounding functions, for studying boundary value problems in the single-valued case. Subsequently, it was generalized by Zanolin [50] and Taddei [48] to the less regular case. We also mention the contributions in $[3,5,20,21,24,25,33,35,36,40,47]$ either for equations or inclusions, but all with rather regular Lyapunov-like functions, that is, at least locally Lipschitzian in $x$.

The authors recently proposed in [10] (see also Remark 2.5) a generalization of this tool for the investigation of globally upper semicontinuous inclusions by means of simply continuous bounding functions.

Recently, Mawhin and Ward Jr. [43] applied a bound set approach to study the periodic boundary value problem for Carathéodory differential equations. As shown in [43] in the single-valued case, when less regularity is assumed with respect to the right-hand side (r.h.s.), some additional conditions must be imposed on the family of bounding functions in order to have a bound set (cf. also [5]).

In Section 2 (see Theorems 2.2, 2.8, and 2.11), we generalize this bound sets theory for the Floquet problem (1.2) when $F$ is a Carathéodory multifunction and the employed bounding functions are less regular. A subsequent discussion (see Remark 2.4) is devoted to the case of $C^{1}$-bounding functions. Moreover, 
comparisons are made with known results in the single-valued case as well as with related results in the framework of the stability theory (see Remark 2.5).

The existence of bounded solutions of the second-order equation

$$
x^{\prime \prime}+f(t, x) x^{\prime}+g(t, x)=0
$$

was extensively investigated both on the positive half line, that is, for $t \in[0,+\infty$, as well as on the entire real line. Several recent results were obtained by means of various methods.

Concerning the investigation on a half line, we refer to the paper of Granas et al. [29], where they combine a sequential approach with the topological transversality theorem for analyzing dynamics in semiconductor theory. We also mention [38], where the existence of a bounded nontrivial solution with an assigned initial point is obtained, for a combustion model, by means of a shooting method. For interesting related earlier papers, see $[22,23]$.

Existence results on the whole real line were found in the Landesman-Lazer case in Ortega and Tineo [45] (see also [1, 26, 42, 44]). We refer, in addition, to the results in [39], proposed for analyzing the waves equation in a reactiondiffusion model and derived with a comparison-type technique. For the results in abstract spaces, see, for example, $[2,32]$.

For systems of equations, recent existence results of bounded solutions were obtained by Mawhin [41] with a similar method as in this paper, when combining a bound sets technique with a convergence result due to Krasnosel'skii [34]; see also $[9,25,42]$.

Note that existence results for bounded solutions of inclusions are rare and they were mostly obtained by means of the viability theory (see, e.g., $[4,6,7,8$, $12,13,25,30]$ and the references therein).

Given a point $x \in \mathbb{R}^{N}$ and a positive constant $r$ throughout the paper, we will denote by $B_{x}^{r}$ the closed ball centered in $x$ and having the radius $r$.

\section{Bound sets for the Floquet boundary value problem}

Consider the Floquet boundary value problem (1.2), where $M$ is a regular $N \times N$ matrix.

Let $\{K(t)\}_{t \in[a, b]}$ be a one-parameter family of nonempty, open, and uniformly bounded subsets of $\mathbb{R}^{N}$ and denote by $\overline{\mathscr{K}}$ and $\partial K$, respectively, the sets

$$
\begin{aligned}
\overline{\mathscr{K}} & =\{(t, x): t \in[a, b], x \in \overline{K(t)}\}, \\
\partial K & =\{(t, x): t \in[a, b], x \in \partial K(t)\} .
\end{aligned}
$$

Definition 2.1. Say that $\{K(t)\}_{t \in[a, b]}$ is a bound set for the boundary value problem (1.2) if there is no solution $x(t)$ of (1.2) such that $x(t) \in \overline{K(t)}$ for all $t \in$ $[a, b]$, and $x(\tau) \in \partial K(\tau)$ for some $\tau \in[a, b]$. 
In this section, we are interested in finding verifiable conditions assuring that $\{K(t)\}_{t \in[a, b]}$ is a bound set for the boundary value problem (1.2). For this aim, we will introduce a family of the so-called bounding functions $\left\{V_{(\tau, \xi)}\right\}_{(\tau, \xi) \in \partial K}$, satisfying suitable transversality conditions. More precisely, given $\tau \in[a, b]$ and $\xi \in \partial K(\tau)$, as usual, by a bounding function, we mean a continuous function $V_{(\tau, \xi)}:[a, b] \times \mathbb{R}^{N} \rightarrow \mathbb{R}$ satisfying

$$
\begin{aligned}
& V_{(\tau, \xi)}(\tau, \xi)=0, \\
& V_{(\tau, \xi)}(t, x) \leq 0, \quad \forall(t, x) \in \overline{\mathscr{K}}, \text { in a neighbourhood of }(\tau, \xi) .
\end{aligned}
$$

We reason by a contradiction, that is, we assume the existence of a solution of (1.2), having the graph entirely contained in $\overline{\mathscr{K}}$ and touching $\partial K$ at some point $(\tau, x(\tau))$. Denoting by $f$ the composed function $V_{(\tau, x(\tau))}(\cdot, x(\cdot))$, we have, according to (2.2), that $f$ is nonpositive in a suitable neighbourhood of the point $\tau$ and $f(\tau)=0$. Assuming various transversality conditions on the bounding functions, we then get various monotonicity properties on $f$ and this yields, in all cases, the required contradiction with (2.2).

In particular, when the multifunction $F$ is globally upper semicontinuous, then a local monotonicity in $\tau$ is sufficient (see Remark 2.5 and [10]). On the contrary, when $F$ is Carathéodory, we need to assure the decreasing monotonicity of $f$ in a left neighbourhood of $\tau$ or the increasing monotonicity in a right one. In conclusion, the theory of bound sets is based on the investigation of local, that is, in one point, or global monotonicity properties of real continuous functions.

Consider, as always in this work, a Carathéodory r.h.s. F. Again, when the bounding functions $V_{(\tau, \xi)}(t, x)$ are more regular, that is, at least locally Lipschitzian in both variables $(t, x)$, a sign condition on their Dini directional derivatives, that is, (2.4), guarantees that $\{K(t)\}_{t \in[a, b]}$ is a bound set. We investigate this situation in Theorem 2.2. On the contrary, in the case of general continuous bounding functions, we need to impose the growth restrictions on $F$, for example, when assuming condition (2.31). We also need a monotonicity result on continuous real functions (see [31, Theorem 5.2.3] and see also [49, Theorem 55.10]) that we reformulate in Lemma 2.7, in our precise framework. With these two ingredients, we are then able to show suitable general transversality conditions, (2.32) and (2.33), on $V_{(\tau, \xi)}$, again assuring that $\{K(t)\}_{t \in[a, b]}$ is a bound set for the Floquet boundary value problem. Such a discussion is contained in Theorem 2.8. Finally, when the growth restriction on $F$ does not depend on $t$, in particular, when $F$ satisfies (2.42), then condition (2.33) can be weakened and the existence of a bound set simply follows by sign conditions on suitable contingent derivatives of $V_{(\tau, \xi)}$, as shown in Theorem 2.11.

As usual in this setting, we introduce the following invariance condition:

$$
M \partial K(a)=\{M \xi: \xi \in \partial K(a)\}=\partial K(b) .
$$


Theorem 2.2. Let $\{K(t)\}_{t \in[a, b]}$ be a family of nonempty, open, and uniformly bounded subsets of $\mathbb{R}^{N}$, satisfying the invariance condition (2.3). Assume that, for every $(\tau, \xi) \in \partial K$, there is a function $V_{(\tau, \xi)}$, locally Lipschitzian in both variables $(t, x)$ and satisfying (2.2).

Suppose, moreover, that for all $\tau \in] a, b], t \leq \tau, x \in \overline{K(t)}$, with $(t, x)$ in a neighbourhood of $(\tau, \xi)$,

$$
\liminf _{h \rightarrow 0^{-}} \frac{V_{(\tau, \xi)}(t+h, x+h w)-V_{(\tau, \xi)}(t, x)}{h}<0, \quad \forall w \in F(t, x) .
$$

Then $\{K(t)\}_{t \in[a, b]}$ is a bound set for (1.2).

Proof. Let $x:[a, b] \rightarrow \mathbb{R}^{N}$ be a solution of (1.2) satisfying $x(t) \in \overline{K(t)}$ for every $t \in[a, b]$, and assume by a contradiction the existence of $\tau \in[a, b]$ such that $x(\tau) \in \partial K(\tau)$.

According to the invariance condition (2.3), $x(a) \in \partial K(a)$ if and only if $x(b) \in$ $\partial K(b)$. Therefore, there is no loss of generality to assume that $\tau \in] a, b]$.

Consider $V_{(\tau, x(\tau))}$ and denote it throughout this proof simply by $V$. By (2.2) and the absolute continuity both of $V$ and of $x$, it follows the existence of $\bar{h}<0$ such that

$$
0 \leq-V(\tau+\bar{h}, x(\tau+\bar{h}))=\int_{\tau+\bar{h}}^{\tau} \frac{d}{d t} V(s, x(s)) d s
$$

Moreover, since $x$ is a solution of (1.1), for almost every $t \in[\tau+\bar{h}, \tau]$, there exist both a function $\Delta(h)$, which in fact depends on $t$, such that $\Delta(h) \rightarrow 0$ when $h \rightarrow 0$, and a vector $w \in F(t, x(t))$ satisfying, for $h$ small enough,

$$
x(t+h)=x(t)+h[w+\Delta(h)]
$$

Hence, the local Lipschitzianity of $V$, combined with (2.4), implies that, for almost every $t \in[\tau+\bar{h}, \tau]$,

$$
\begin{aligned}
\frac{d}{d t} V(t, x(t)) & =\lim _{h \rightarrow 0^{-}} \frac{V(t+h, x(t)+h[w+\Delta(h)])-V(t, x(t))}{h} \\
& =\liminf _{h \rightarrow 0^{-}} \frac{V(t+h, x(t)+h[w+\Delta(h)])-V(t, x(t))}{h} \\
& \leq \liminf _{h \rightarrow 0^{-}}\left[\frac{V(t+h, x(t)+h w)-V(t, x(t))}{h}+L_{(\tau, x(\tau))}|\Delta(h)|\right] \\
& <0,
\end{aligned}
$$

and this leads to a contradiction with (2.5). 
Remark 2.3. For $\tau<b$, the conclusion of Theorem 2.2 also holds when replacing assumption (2.4) by

$$
\limsup _{h \rightarrow 0^{+}} \frac{V_{(\tau, \xi)}(t+h, x+h w)-V_{(\tau, \xi)}(t, x)}{h}>0, \quad \forall w \in F(t, x),
$$

for all $t \geq \tau$ and $x \in \overline{K(t)}$, with $(t, x)$ in a neighbourhood of $(\tau, \xi)$. It is in fact possible to prove, similarly as in the previous theorem, that (2.8) implies the increasing monotonicity of $V_{(\tau, x(\tau))}(\cdot, x(\cdot))$ in a right neighbourhood of $\tau$.

Remark 2.4. Notice that when $V_{(\tau, \xi)}$ is a function from the class $C^{1}$, then both (2.4) and (2.8) reduce to

$$
\left(\nabla V_{(\tau, \xi)}(t, x),(1, w)\right) \neq 0, \quad \forall w \in F(t, x)
$$

In [43, Theorem 2.1], an existence result for the periodic boundary value problem associated to a Carathéodory differential equation is given by means of a $C^{1}$-guiding-like function. They consider an autonomous bound set defined as the counter image of the negative real line through a unique bounding function $V: \mathbb{R}^{N} \rightarrow \mathbb{R}$ from the class $C^{1}$ and they ask $V$ to satisfy the following condition:

$$
(\nabla V(x), f(t, x))<0, \quad \forall(t, x) \in[a, b] \times V^{-1}([-\epsilon, 0])
$$

where $\epsilon$ is a positive constant.

Since we can reformulate $(2.9)$ as $(\nabla V(x), w) \neq 0$ for all $t \in] a, b], x \in \bar{K}$ in a neighbourhood of $\partial K$, and $w \in F(t, x),(2.9)$ becomes trivially a generalization of (2.10) to the multivalued case and to nonautonomous bound sets defined by means of a family of bounding functions, instead of a guiding function.

Remark 2.5. In [10], the authors prove the existence of a bound set for the Floquet problem (1.2) when $F$ is globally upper semicontinuous. In Remark 3 of the quoted paper, a bound set is obtained with assumption (2.4) of Theorem 2.2, replaced by

$$
0 \notin\left[\liminf _{h \rightarrow 0^{+}} \frac{V_{(\tau, \xi)}\left(\tau+h, \xi+h w_{1}\right)}{h}, \limsup _{h \rightarrow 0^{-}} \frac{V_{(\tau, \xi)}\left(\tau+h, \xi+h w_{2}\right)}{h}\right],
$$

for all $\tau \in] a, b\left[, \xi \in \partial K(\tau)\right.$, and $w_{1}, w_{2} \in F(\tau, \xi)$, and by

$$
0 \notin\left[\liminf _{h \rightarrow 0^{+}} \frac{V_{(a, \xi)}\left(a+h, \xi+h w_{a}\right)}{h}, \limsup _{h \rightarrow 0^{-}} \frac{V_{(b, M \xi)}\left(b+h, M \xi+h w_{b}\right)}{h}\right],
$$

for all $\xi \in \partial K(a), w_{a} \in F(a, \xi)$, and $w_{b} \in F(b, M \xi)$. 
In order to make a comparison of the above conditions in [10] with those in Theorem 2.2, we should point out that, unlike in Theorem 2.2, the above conditions are only assumed to hold in one point. On the other hand, if conditions (2.4) and (2.8) are localized into one point, then they are implied by their analogues in [10].

Locally Lipschitzian Lyapunov functions were used in [25, Proposition 14.1] to obtain necessary and sufficient conditions for the strong stability of the zero solution of a differential inclusion. For this aim, condition (2.4) is globally assumed to guarantee the decreasing monotonicity of the Lyapunov functions along all the solutions of the inclusion.

The same problem was recently analyzed by Bacciotti et al. [15] for autonomous continuous inclusions, possibly Lipschitz continuous. They provide a characterization of the monotonicity of a Lyapunov function along all the trajectories, both in terms of proximal subdifferential and of contingent derivatives.

Example 2.6. Consider the antiperiodic problem

$$
\begin{aligned}
x^{\prime} & \in F(t, x), \\
x(b) & =-x(a),
\end{aligned}
$$

where $F:[a, b] \times \mathbb{R}^{N} \rightsquigarrow \mathbb{R}^{N}$ is a Carathéodory multifunction.

Assume that there exist positive constants $\epsilon$ and $R_{j}, j=1, \ldots, N$, such that, denoted by

$$
\left.K=\Pi_{j=1}^{N}\right]-R_{j}, R_{j}[
$$

and by

$$
\partial K_{j}=\left\{\xi \in \partial K: \xi_{j}= \pm R_{j}\right\}
$$

one has, for all $j=1, \ldots, N, \xi \in \partial K_{j}, x \in \bar{K} \cap B_{\xi}^{\epsilon}, t>a$, and $w \in F(t, x)$, ( $\operatorname{sign} \xi_{j}$. $\left.w_{j}\right) \neq 0$. Consider for every $j=1, \ldots, N$ and $\xi \in \partial K_{j}$, the $C^{1}$-function defined by $V_{\xi}(x)=\operatorname{sign} \xi_{j} \cdot x_{j}-R_{j}$. We have $V_{\xi}(\xi)=0, V_{\xi}(x) \leq 0$, for all $x \in \bar{K}$. Moreover, $\nabla V_{\xi}(x) \equiv \operatorname{sign} \xi_{j} \cdot e_{j}$. Hence, for every $j=1, \ldots, N, \xi \in \partial K_{j}, x \in \bar{K} \cap B_{\xi}^{\epsilon}, t>a$, and $w \in F(t, x)$,

$$
\left(\nabla V_{\xi}(x), w\right)=\operatorname{sign} \xi_{j} \cdot w_{j} \neq 0
$$

Therefore, recalling also Remark $2.4, K$ satisfies all the hypotheses to be a bound set for (2.13), because (2.3) is equivalent to the symmetry of $\partial K$ with respect to the origin.

We propose now a general theory on bound sets valid for arbitrary continuous bounding functions. The following theorem, involving Dini derivatives, is an appropriate version of a known result (see [31, Theorem 5.2.3], see also [49, Theorem 55.10]) on monotonicity properties for real continuous functions. 
For this purpose, given a continuous function $f:[a, b] \rightarrow \mathbb{R}$ and a point $t_{0} \in$ ]$a, b[$, we denote by

$$
\begin{aligned}
& D_{-} f\left(t_{0}\right)=\liminf _{h \rightarrow 0^{-}} \frac{f\left(t_{0}+h\right)-f\left(t_{0}\right)}{h}, \\
& D^{-} f\left(t_{0}\right)=\limsup _{h \rightarrow 0^{-}} \frac{f\left(t_{0}+h\right)-f\left(t_{0}\right)}{h},
\end{aligned}
$$

respectively, the lower and upper left Dini derivatives in $t_{0}$. Similarly, letting $h \rightarrow 0^{+}$, we obtain the right Dini derivatives $D_{+} f\left(t_{0}\right)$ and $D^{+} f\left(t_{0}\right)$ of $f$ in $t_{0}$.

Lemma 2.7. Let $f:[a, b] \rightarrow \mathbb{R}$ be a continuous function such that

$$
\begin{aligned}
& D_{-} f(t)<0, \quad \text { for a.a. } t \\
& D_{-} f(t)<+\infty, \quad \forall t \text { except at most a countable set. }
\end{aligned}
$$

Then $f$ is monotone decreasing in $[a, b]$.

Proof. Consider a positive real number $\epsilon$ and take $n \in \mathbb{N}$. Then (2.18) implies the existence of an open subset $Z_{n}^{\epsilon}$ of $[a, b]$ such that

$$
\left\{t \in[a, b]: D_{-} f(t) \geq 0\right\} \subset Z_{n}^{\epsilon}, \quad \lambda\left(Z_{n}^{\epsilon}\right)<\frac{\epsilon}{2^{n}},
$$

where $\lambda$ denotes the Lebesgue measure on $[a, b]$. The properties of $\lambda$ yield, for every $t \in[a, b]$,

$$
\sum_{n=1}^{+\infty} \lambda\left([t, b] \cap Z_{n}^{\epsilon}\right) \leq \sum_{n=1}^{+\infty} \lambda\left(Z_{n}^{\epsilon}\right)<\sum_{n=1}^{+\infty} \frac{\epsilon}{2^{n}}=\epsilon
$$

Therefore, the function

$$
\begin{aligned}
Z:[a, b] & \longrightarrow \mathbb{R} \\
t & \longrightarrow f(t)+\sum_{n=1}^{+\infty} \lambda\left([t, b] \cap Z_{n}^{\epsilon}\right)
\end{aligned}
$$

is well defined. Given $\delta>0$, take $\bar{n}$ such that

$$
\sum_{n=\bar{n}+1}^{+\infty} \lambda\left(Z_{n}^{\epsilon}\right) \leq \frac{\delta}{2}
$$


For every $t<t^{\prime}$, with $t^{\prime}-t \leq \delta / 2 \bar{n}$, by the properties of the Lebesgue measure, it follows that

$$
\begin{aligned}
0 & \leq \sum_{n=1}^{+\infty} \lambda\left([t, b] \cap Z_{n}^{\epsilon}\right)-\sum_{n=1}^{+\infty} \lambda\left(\left[t^{\prime}, b\right] \cap Z_{n}^{\epsilon}\right)=\sum_{n=1}^{+\infty} \lambda\left(\left[t, t^{\prime}\right] \cap Z_{n}^{\epsilon}\right) \\
& =\sum_{n=1}^{\bar{n}} \lambda\left(\left[t, t^{\prime}\right] \cap Z_{n}^{\epsilon}\right)+\sum_{n=\bar{n}+1}^{+\infty} \lambda\left(\left[t, t^{\prime}\right] \cap Z_{n}^{\epsilon}\right) \leq \bar{n}\left(t^{\prime}-t\right)+\frac{\delta}{2} \leq \delta .
\end{aligned}
$$

We have so proved that

$$
\left|\sum_{n=1}^{+\infty} \lambda\left([t, b] \cap Z_{n}^{\epsilon}\right)-\sum_{n=1}^{+\infty} \lambda\left(\left[t^{\prime}, b\right] \cap Z_{n}^{\epsilon}\right)\right| \leq \delta, \quad \text { whenever }\left|t-t^{\prime}\right| \leq \frac{\delta}{2 \bar{n}}
$$

Consequently, the function $t \rightarrow \sum_{n=1}^{+\infty} \lambda\left([t, b] \cap Z_{n}^{\epsilon}\right)$ is uniformly continuous on $[a, b]$ and, because of the continuity of $f$, this implies the continuity of $Z$.

Since, by the definition, $t \in Z_{n}^{\epsilon}$, for every $t$ such that $D_{-} f(t) \geq 0$, and $Z_{n}^{\epsilon}$ is open, it follows that, for every $n \in \mathbb{N}$, there exists $h_{n}<0$ such that $\left[t+h_{n}, t\right] \subset Z_{n}^{\epsilon}$ and $h_{n} \rightarrow 0^{-}$when $n \rightarrow+\infty$.

For every $n \in \mathbb{N}$, define $\rho_{n}=\max _{i=1, \ldots, n} h_{i}$ and take $h \in\left[\rho_{n}, 0[\right.$. It follows that

$$
\begin{aligned}
& \frac{\sum_{i=1}^{+\infty} \lambda\left([t+h, b] \cap Z_{i}^{\epsilon}\right)-\sum_{i=1}^{+\infty} \lambda\left([t, b] \cap Z_{i}^{\epsilon}\right)}{h} \\
&=\sum_{i=1}^{+\infty} \frac{\lambda\left([t+h, t] \cap Z_{i}^{\epsilon}\right)}{h} \leq \sum_{i=1}^{n} \frac{\lambda([t+h, t])}{h}=-n .
\end{aligned}
$$

Therefore, since $n$ is arbitrary, we have that

$$
\frac{d}{d t} \sum_{i=1}^{+\infty} \lambda\left([t, b] \cap Z_{n}^{\epsilon}\right)(t)=-\infty, \quad \forall t \in[a, b]: D_{-} f(t) \geq 0
$$

Hence, according to condition (2.19),

$$
D_{-} Z(t)=-\infty, \quad \forall t \in[a, b]: 0 \leq D_{-} f(t)<+\infty \text {. }
$$

Moreover, since the function $t \rightarrow \sum_{n=1}^{+\infty} \lambda\left([t, b] \cap Z_{n}^{\epsilon}\right)$ is monotone nonincreasing, recalling (2.19), it follows that $D_{-} Z(t)<0$ for all $t \in[a, b]$ with the possible exception of a countable set, implying that $\left\{Z(t): t \in[a, b], D_{-} Z(t) \geq 0\right\}$ is at most countable.

Now suppose that there exists $t_{1}<t_{2}$ satisfying $Z\left(t_{1}\right)<Z\left(t_{2}\right)$. Since $Z$ is continuous, the interval $\left[Z\left(t_{1}\right), Z\left(t_{2}\right)\right]$ contains a continuum of points. Hence, it is 
then possible to find $r \in\left[Z\left(t_{1}\right), Z\left(t_{2}\right)\right]$ and $\bar{t} \in\left[t_{1}, t_{2}\right]$ such that $\bar{t}=\min \{t \in$ $\left.\left[t_{1}, t_{2}\right]: Z(t)=r\right\}$,

$$
D \_Z(\bar{t})<0 \text {. }
$$

Again, by the continuity of $Z$, it follows that $Z(t)<r, \forall t \in\left[t_{1}, \bar{t}\right]$, that is, $D_{-} Z(\bar{t})$ $\geq 0$, which is a contradiction with (2.29).

Thus, $Z$ is monotone nonincreasing, which yields, for every $t_{1}<t_{2}$ and every $\epsilon>0$,

$$
\begin{aligned}
f\left(t_{1}\right)-f\left(t_{2}\right) & \geq \sum_{n=1}^{+\infty} \lambda\left(\left[t_{2}, b\right] \cap Z_{n}^{\epsilon}\right)-\sum_{n=1}^{+\infty} \lambda\left(\left[t_{1}, b\right] \cap Z_{n}^{\epsilon}\right) \\
& =-\sum_{n=1}^{+\infty} \lambda\left(\left[t_{1}, t_{2}\right] \cap Z_{n}^{\epsilon}\right) \geq-\sum_{n=1}^{+\infty} \lambda\left(Z_{n}^{\epsilon}\right)=-\epsilon,
\end{aligned}
$$

implying that $f$ is monotone nonincreasing.

Finally, if there exists $t_{1}<t_{2}$ such that $f\left(t_{1}\right)=f\left(t_{2}\right)$, then $f$ is constant in $\left[t_{1}, t_{2}\right]$ which contradicts (2.5), and so the proof is complete.

We are now able to state our main result concerning the existence of bound sets defined by means of continuous bounding functions.

For this aim, we need to assume certain growth conditions on the multifunction $F$, namely, the existence of $c \in L^{1}([a, b])$ such that

$$
|F(t, x)| \leq c(t), \quad \forall(t, x) \in \overline{\mathscr{K}}
$$

Theorem 2.8. Let $\{K(t)\}_{t \in[a, b]}$ be a family of nonempty and open subsets of $\mathbb{R}^{N}$, uniformly bounded by $B_{0}^{R}$ and satisfying the invariance condition (2.3). Assume that, for every $(\tau, \xi) \in \partial K$, there is a continuous function $V_{(\tau, \xi)}$ satisfying (2.2).

Suppose, moreover, that for all $\tau>a, t \leq \tau$, and $x \in \overline{K(t)}$, with $(t, x)$ in a neighbourhood of $(\tau, \xi)$,

$$
\begin{aligned}
& \limsup _{\substack{h \rightarrow 0^{-} \\
v \rightarrow w}} \frac{V_{(\tau, \xi)}(t+h, x+h v)-V_{(\tau, \xi)}(t, x)}{h}<0, \quad \forall w \in F(t, x), \\
& \limsup _{\substack{h \rightarrow 0^{-} \\
y \rightarrow x}} \frac{V_{(\tau, \xi)}(t+h, y)-V_{(\tau, \xi)}(t, x)}{h}<+\infty . \\
& |y-x| \leq \int_{t+h}^{t} c(s) d s
\end{aligned}
$$

Then $\{K(t)\}_{t \in[a, b]}$ is a bound set for (1.2) provided (2.31) holds.

Proof. Reasoning as in Theorem 2.2, if we suppose that there exists a solution $x$ of (1.2) whose graph is entirely contained in $\overline{\mathscr{K}}$ and touches its boundary in $\tau$, we can assume, without any loss of generality, that $\tau \in] a, b]$. Take $V_{(\tau, x(\tau))}$ and, 
for the sake of simplicity, denote it by $V$. From (2.32) and (2.6), we get

$$
\begin{aligned}
& \limsup _{h \rightarrow 0^{-}} \frac{V(t+h, x(t+h))-V(t, x(t))}{h} \\
& \quad \limsup _{h \rightarrow 0^{-}} \frac{V(t+h, x(t)+h(w+\Delta(h)))-V(t, x(t))}{h} \\
& \quad \leq \limsup _{\substack{h \rightarrow 0^{-} \\
v \rightarrow w}} \frac{V(t+h, x(t)+h v)-V(t, x(t))}{h}<0,
\end{aligned}
$$

for a.a. $t \in[\tau+\bar{h}, \tau]$ with $\bar{h}<0$ sufficiently small, implying

$$
D^{-} V(t, x(t))<0, \quad \text { for a.e. } t \in[\tau+\bar{h}, \tau] \text {. }
$$

Moreover, since $x$ is a solution of (1.2) and $x(t) \in K(t)$, for all $t \in[\tau+\bar{h}, \tau]$ and $h$ small enough, it follows that

$$
|x(t+h)-x(t)| \leq \int_{t+h}^{t}\left|x^{\prime}(s)\right| d s \leq \int_{t+h}^{t} c(s) d s
$$

Thus,

$$
\begin{aligned}
& \limsup _{h \rightarrow 0^{-}} \frac{V(t+h, x(t+h))-V(t, x(t))}{h} \\
& \leq \limsup _{\substack{h \rightarrow 0^{-} \\
y \rightarrow x \\
|y-x| \leq \int_{t+h}^{t} c}} \frac{V(s) d s}{h}<+\infty,
\end{aligned}
$$

because of (2.33).

We have so proved that $D^{-} V(t, x(t))<+\infty, \forall t \in[\tau+\bar{h}, \tau]$. Hence, Lemma 2.7 implies the decreasing monotonicity of $t \rightarrow V(t, x(t))$ in a left neighbourhood of $\tau$ which leads to a contradiction with (2.2). Therefore, $x(t) \in K(t)$ for all $t \in[a, b]$.

Remark 2.9. Taken $\tau<b$, the conclusion of Theorem 2.8 holds true also if we replace assumptions (2.32) and (2.33) by

$$
\begin{aligned}
& \liminf _{\substack{h \rightarrow 0^{+} \\
v \rightarrow w}} \frac{V_{(\tau, \xi)}(t+h, x+h v)-V_{(\tau, \xi)}(t, x)}{h}>0, \quad \forall w \in F(t, x), \\
& \liminf _{\substack{h \rightarrow 0^{+} \\
y \rightarrow x \\
-x \mid \leq \int_{t+h}^{t} c(s) d s}} \frac{V_{(\tau, \xi)}(t+h, y)-V_{(\tau, \xi)}(t, x)}{h}>-\infty,
\end{aligned}
$$


for all $t \geq \tau$ and $x \in \overline{K(t)}$, with $(t, x)$ in a neighbourhood of $(\tau, \xi)$. It is in fact sufficient to make use of the increasing monotonicity of $t \rightarrow V_{(\tau, x(\tau))}(t, x(t))$ in a right neighbourhood of $\tau$.

Remark 2.10. Observe that if $V_{(\tau, \xi)}$ is locally Lipschitzian in both variables $(t, x)$, it is possible to prove, as in [10, Remark 3], that (2.4) and (2.8) are, respectively, weaker than (2.32) and (2.38). Moreover, (2.33) and (2.39) are not necessary to get a bound set, as Theorem 2.2 shows.

In [10, Theorem 3], a bound set is obtained without requiring hypothesis (2.33) and with assumption (2.32) replaced by the conditions

$$
0 \notin\left[\liminf _{\substack{v \rightarrow W_{1} \\ h \rightarrow 0^{+}}} \frac{V_{(\tau, \xi)}(\tau+h, \xi+h v)}{h}, \limsup _{\substack{v \rightarrow w_{2} \\ h \rightarrow 0^{-}}} \frac{V_{(\tau, \xi)}(\tau+h, \xi+h v)}{h}\right],
$$

for all $\tau \in] a, b\left[, \xi \in \partial K(\tau)\right.$, and $w_{1}, w_{2} \in F(\tau, \xi)$, and

$$
0 \notin\left[\liminf _{\substack{v \rightarrow w_{a} \\ h \rightarrow 0^{+}}} \frac{V_{(a, \xi)}(a+h, \xi+h v)}{h}, \limsup _{\substack{v \rightarrow w_{b} \\ h \rightarrow 0^{-}}} \frac{V_{(b, M \xi)}(b+h, M \xi+h v)}{h}\right],
$$

for all $\xi \in \partial K(a), w_{a} \in F(a, \xi)$, and $w_{b} \in F(b, M \xi)$.

Notice that both (2.32) and (2.38) imply the above assumptions. Hence, in the globally upper semicontinuous case, conditions weaker than those required in Theorem 2.8 allow us to get the existence of a bound set.

If the growth conditions imposed on $F$ do not depend on $t$; precisely, and if there exists a real constant $c$ such that

$$
|F(t, x)| \leq c, \quad \forall(t, x) \in[a, b] \times \overline{\mathscr{K}}
$$

then (2.33) can be weakened as shown by the following result.

THeOREM 2.11. Let $\{K(t)\}_{t \in[a, b]}$ be a family of nonempty and open subsets of $\mathbb{R}^{N}$, uniformly bounded by $B_{0}^{R}$ and satisfying the invariance condition (2.3). Assume that, for every $(\tau, \xi) \in \partial K$, there is a continuous function $V_{(\tau, \xi)}$ satisfying (2.2).

Suppose, moreover, that for all $\tau>a, t \leq \tau$, and $x \in \overline{K(t)}$, with $(t, x)$ in a neighbourhood of $(\tau, \xi),(2.32)$ holds jointly with

$$
\limsup _{\substack{h \rightarrow 0^{-} \\ v \rightarrow w}} \frac{V_{(\tau, \xi)}(t+h, x+h v)-V_{(\tau, \xi)}(t, x)}{h}<+\infty, \quad \forall w \in B_{0}^{c} .
$$

Then $\{K(t)\}_{t \in[a, b]}$ is a bound set for (1.2). 
Proof. Reasoning as in the proof of Theorem 2.8, if $x$ is a solution of (1.2) whose graph entirely lays in $\overline{\mathscr{K}}$ and touches its boundary in $\tau \in] a, b]$, we get a sufficiently small negative $\bar{h}$ such that

$$
D^{-} V_{(\tau, x(\tau))}(t, x(t))<0, \quad \text { for a.a. } t \in[\tau+\bar{h}, \tau]
$$

Moreover, according to the growth condition (2.42), it holds that $\left|x^{\prime}(t)\right| \leq c$, implying that $x$ is a Lipschitzian function with the Lipschitz constant $c$.

Take $t \in[\tau+\bar{h}, \tau],\left\{h_{n}\right\}_{n \in \mathbb{N}} \subset\left[\tau-t+\bar{h}, 0\left[\right.\right.$ with $h_{n} \rightarrow 0^{-}$, and consider the sequence

$$
\left\{\frac{x\left(t+h_{n}\right)-x(t)}{h_{n}}\right\}_{n \in \mathbb{N}} .
$$

Since every bounded sequence has a convergent subsequence, again denoted as the sequence, there exist $\left\{\Delta_{n}\right\}_{n \in \mathbb{N}}$, with $\Delta_{n} \rightarrow 0$ as $n \rightarrow+\infty$, and $w \in B_{0}^{c}$ such that

$$
x\left(t+h_{n}\right)=x(t)+h_{n}\left[w+\Delta_{n}\right], \quad \forall n \in \mathbb{N} .
$$

Therefore, (2.43) yields

$$
\begin{aligned}
\limsup _{n \rightarrow+\infty} & \frac{V_{(\tau, x(\tau))}\left(t+h_{n}, x\left(t+h_{n}\right)\right)-V_{(\tau, x(\tau))}(t, x(t))}{h_{n}} \\
\quad \leq & \limsup _{\substack{h \rightarrow 0^{-} \\
v \rightarrow w}} \frac{V_{(\tau, x(\tau))}(t+h, x(t)+h v)-V_{(\tau, x(\tau))}(t, x(t))}{h}<+\infty,
\end{aligned}
$$

that is,

$$
D^{-} V_{(\tau, x(\tau))}(t, x(t))<+\infty, \quad \forall t \in[\tau+\bar{h}, \tau]
$$

Hence, Lemma 2.7 implies the decreasing monotonicity of $t \rightarrow V_{(\tau, x(\tau))}(t, x(t))$ in $[\tau+\bar{h}, \tau]$ which leads to a contradiction with (2.2). Therefore, $x(t) \in K(t)$ for all $t \in[a, b]$.

Remark 2.12. Observe that condition (2.33) is, under hypothesis (2.42), equivalent to

$$
\limsup _{\substack{(h, v) \rightarrow\left(0^{-}, x\right) \\|y-x| \leq h c}} \frac{V_{(\tau, \xi)}(t+h, y)-V_{(\tau, \xi)}(t, x)}{h}<+\infty .
$$


Therefore, since, for every $w \in B_{0}^{c}$,

$$
\begin{gathered}
\underset{\substack{h \rightarrow 0^{-} \\
v \rightarrow w}}{\limsup } \frac{V_{(\tau, \xi)}(t+h, x+h v)-V_{(\tau, \xi)}(t, x)}{h} \\
\quad \limsup _{\substack{h \rightarrow 0^{-} \\
y \rightarrow x \\
y-x \mid \leq h c}} \frac{V_{(\tau, \xi)}(t+h, y)-V_{(\tau, \xi)}(t, x)}{h},
\end{gathered}
$$

the previous theorem shows that if we assume a stronger condition on the growth behaviour of the multifunction, it is possible to get the existence of a bound set by means of weaker assumptions on the bounding functions.

Remark 2.13. Notice that the conclusion of Theorem 2.11 also holds if we replace (2.32) and (2.43) by (2.38) and

$$
\liminf _{\substack{h \rightarrow 0^{+} \\ v \rightarrow w}} \frac{V_{(\tau, \xi)}(t+h, x+h v)-V_{(\tau, \xi)}(t, x)}{h}>-\infty, \quad \forall w \in B_{0}^{c} .
$$

Moreover, if $V_{(\tau, \xi)}$ is locally Lipschitzian in $(t, x)$, with the Lipschitz constant $L$, for $w \in B_{0}^{c}$ and $v \in B_{w}^{1}$, then one has that

$$
\left|\frac{V_{(\tau, \xi)}(t+h, x+h v)-V_{(\tau, \xi)}(t, x)}{h}\right| \leq L|(1, v)| \leq L(1+|v|) \leq L(2+c) .
$$

Hence, all the contingent derivatives are finite and, in particular, condition (2.43) trivially holds.

Nevertheless, in the locally Lipschitzian case, the conditions of Theorem 2.2 are weaker than those required in the previous theorem (see Remark 2.10).

If $V_{(\tau, \xi)}$ is only locally Lipschitzian in $x$, uniformly with respect to $t$, then (2.43) or (2.51) reduces to

$$
\limsup _{h \rightarrow 0^{-}} \frac{V_{(\tau, \xi)}(t+h, x)-V_{(\tau, \xi)}(t, x)}{h}<+\infty
$$

or

$$
\liminf _{h \rightarrow 0^{+}} \frac{V_{(\tau, \xi)}(t+h, x)-V_{(\tau, \xi)}(t, x)}{h}>-\infty
$$

Thus, (2.43) or (2.51) is implied by (2.32) or (2.38), respectively.

\section{Solutions of the Floquet boundary value problem}

Now, we consider the Floquet boundary value problem

$$
\begin{gathered}
x^{\prime}+A(t) x \in F(t, x), \quad \text { for a.a. } t \in[a, b], \\
x(b)=M x(a),
\end{gathered}
$$


where $F:[a, b] \times \mathbb{R}^{N} \rightsquigarrow \mathbb{R}^{N}$ is a Carathéodory multifunction with nonempty, compact, and convex values, $A:[a, b] \rightarrow \mathbb{R}^{N} \times \mathbb{R}^{N}$ is a continuous $N \times N$ matrix, and $M$ is a regular $N \times N$ matrix.

In this section, we combine the bound sets approach, developed in the previous one, with the following appropriate modification of a continuation principle by Andres et al. (see [7, Theorem 2.33]) in order to solve (3.1).

Proposition 3.1 (see [5, Theorem 1]). Consider the boundary value problem

$$
\begin{gathered}
x^{\prime} \in F(t, x), \quad \text { for a.a. } t \in[a, b], \\
x \in S,
\end{gathered}
$$

where $F:[a, b] \times \mathbb{R}^{N} \rightsquigarrow \mathbb{R}^{N}$ is a Carathéodory multifunction with nonempty, compact, and convex values and $S \subset \mathrm{AC}\left([a, b], \mathbb{R}^{N}\right)$.

Let $G:[a, b] \times \mathbb{R}^{N} \times \mathbb{R}^{N} \times[0,1] \rightsquigarrow \mathbb{R}^{N}$ be a Carathéodory map, with nonempty, compact, and convex values such that

$$
G(t, p, p, 1) \subset F(t, p), \quad \forall(t, p) \in[a, b] \times \mathbb{R}^{N}
$$

Assume that

(1) there exist a bounded retract $Q$ of $C\left([a, b], \mathbb{R}^{N}\right)$ such that $Q \backslash \partial Q$ is nonempty and a closed bounded subset $S_{1}$ of $S$ such that the associated problem

$$
\begin{gathered}
x^{\prime} \in G(t, x, q(t), \lambda), \quad \text { for a.a. } t \in[a, b], \\
x \in S_{1},
\end{gathered}
$$

is solvable with $R_{\delta}$-sets (for the definition, see, e.g., [30]) of solutions, for $\operatorname{each}(q, \lambda) \in Q \times[0,1]$;

(2) there exists an integrable function $c:[a, b] \rightarrow \mathbb{R}$ such that

$$
|G(t, x(t), q(t), \lambda)| \leq c(t), \quad \text { a.e. in }[a, b],
$$

for any $(q, \lambda, x) \in \Gamma_{T}$, where $T$ denotes the map which assigns to any $(q, \lambda) \in$ $Q \times[0,1]$ the set of solutions of (3.4) and $\Gamma_{T}$ its graph;

(3) $T(Q \times\{0\}) \subset Q$;

(4) the map $T$ has no fixed points on the boundary $\partial Q$ of $Q$, for every $(q, \lambda) \in$ $Q \times[0,1]$.

Then (3.2) admits a solution.

As usual in this setting, we will proceed by the following way to construct the set $Q$,

$$
Q=\left\{q \in C\left([a, b], \mathbb{R}^{N}\right): q(t) \in \overline{K(t)}, \forall t \in[a, b]\right\}
$$


We recall [10, Proposition 2] for sufficient conditions in order that $Q$ is a retract of $C\left([a, b], \mathbb{R}^{N}\right)$.

We are now able to state a result about the solvability of (3.1).

Theorem 3.2. Consider the Floquet boundary value problem (3.1).

Assume that

(1) the associated homogeneous problem

$$
\begin{aligned}
x^{\prime}+A(t) x & =0, \quad \text { for a.a. } t \in[a, b], \\
x(b) & =M x(a)
\end{aligned}
$$

has only the trivial solution;

(2) there exists a Carathéodory multifunction $G:[a, b] \times \mathbb{R}^{N} \times \mathbb{R}^{N} \times[0,1] \rightsquigarrow$ $\mathbb{R}^{N}$ with nonempty, compact, and convex values such that

$$
G(t, p, p, 1) \subset F(t, p), \quad \forall(t, p) \in[a, b] \times \mathbb{R}^{N} ;
$$

(3) there exists a family $\{K(t)\}_{t \in[a, b]}$ of nonempty and open subsets of $\mathbb{R}^{N}$, uniformly bounded by $B_{0}^{R}$, such that $\overline{\mathscr{T}}$ is a retract of $[a, b] \times \mathbb{R}^{N}$ with a retraction $\phi:[a, b] \times \mathbb{R}^{N} \rightarrow \overline{\mathscr{Y}}$ which is the identity on its first component, that is, $\phi(t, x)=(t, \tilde{\phi}(t, x))$ and $\partial K$ is closed;

(4) $G(t, \cdot, q, \lambda)$ is Lipschitzian with a sufficiently small Lipschitz constant, for each $(t, \lambda) \in[a, b] \times[0,1]$ and $q \in \bar{K}(t)$;

(5) there exists an integrable function $c:[a, b] \rightarrow \mathbb{R}$ such that

$$
|G(t, x(t), q(t), \lambda)| \leq c(t), \quad \text { a.e. in }[a, b]
$$

for any $(q, \lambda, x) \in \Gamma_{T}$, where $Q=\left\{q \in C\left([a, b], \mathbb{R}^{N}\right): q(t) \in \overline{K(t)}\right.$, for all $t \in$ $[a, b]\}, T$ denotes the map which assigns to any $(q, \lambda) \in Q \times[0,1]$ the set of solutions of

$$
\begin{aligned}
x^{\prime}+A(t) x & \in G(t, x, q(t), \lambda), \quad \text { for a.a. } t \in[a, b], \\
x(b) & =M x(a),
\end{aligned}
$$

and $\Gamma_{T}$ its graph;

(6) $T(Q \times\{0\}) \subset Q$ and $\partial Q$ is fixed point free, that is, $\{q \in Q: q \in T(q, 0)\} \cap$ $\partial Q=\varnothing ;$

(7) for every $(\tau, \xi) \in \partial K$, there exists a function $V_{(\tau, \xi)}:[a, b] \times \mathbb{R}^{N} \rightarrow \mathbb{R}$, locally Lipschitzian in $(t, x)$ and satisfying (2.2);

(8) for every $\tau \in] a, b], \xi \in \partial K(\tau), t \leq \tau, x \in \overline{K(t)}$, with $(t, x)$ in a neighbourhood of $(\tau, \xi),(q, \lambda) \in Q \times] 0,1]$, one has

$\liminf _{h \rightarrow 0^{-}} \frac{V_{(\tau, \xi)}(t+h, x+h w)}{h}<0, \quad \forall w \in G(t, x, q(t), \lambda)-A(t) x ;$

(9) $M \partial K(a)=\{M \xi: \xi \in \partial K(a)\}=\partial K(b)$.

Then (3.1) admits a solution. 
Proof. Define $Q$ as in (5). Hence, $Q$ is a closed, bounded subset of $C\left([a, b], \mathbb{R}^{N}\right)$. From assumption (3) and [10, Proposition 2], we get that $Q$ is a bounded retract of $C\left([a, b], \mathbb{R}^{N}\right)$. In addition, since $\partial K$ is closed, we can reason as in the proof of [10, Theorem 4] and obtain that $q \in \partial Q$ if and only if $q(\bar{t}) \in \partial K(\bar{t})$, for some $\bar{t} \in[a, b]$. Thus, $Q \backslash \partial Q$ is nonempty.

According to conditions (1), (2), and (4), we are able to apply [14, Theorem 4] to problem (3.10), with $q \in Q$ and $\lambda \in[0,1]$, in order to assure its solvability with an $R_{\delta}$-set of solutions. In particular, each solution set lies in some ball $B_{0}^{\rho}$. Moreover, it follows from the proof of [14, Theorem 4] that $B_{0}^{\rho}$ can be taken the same for all $q \in Q$. Therefore, defining

$$
S_{1}=B_{0}^{\rho} \cap\left\{x \in \operatorname{AC}\left([a, b], \mathbb{R}^{N}\right): x(b)=M x(a)\right\}
$$

we obtain that $S_{1}$ is bounded.

Thanks to Theorem 2.2, assumptions (7), (8), and (9) guarantee that $\{K(t)\}_{t \in[a, b]}$ is a bound set for each problem (3.10) with $q \in Q$ and $\left.\left.\lambda \in\right] 0,1\right]$. Hence, any solution $x \in Q$ of (3.10) satisfies $x(t) \in K(t)$, for all $t \in[a, b]$, so $x \notin \partial Q$. Therefore, $T$ is fixed-point free on $\partial Q$, for all $\lambda \neq 0$. By assumption (6), $T$ is also fixed-point free on $\partial Q$, for $\lambda=0$.

In conclusion, all the requirements of Proposition 3.1 are satisfied and (3.1) has a solution $x$ such that $x(t) \in \bar{K}(t)$, for each $t \in[a, b]$.

Remark 3.3. Since all the sets $K(t)$ are uniformly bounded, in the previous theorem, we can replace assumption (5) with the following one:

$$
|G(t, x(t), q(t), \lambda)| \leq d(t)(1+|x(t)|),
$$

for all $t \in[a, b],(q, \lambda, x) \in \Gamma_{T}$, and with $d:[a, b] \rightarrow \mathbb{R}$ integrable.

Similar versions of Theorem 3.2 hold when we consider continuous bounding functions. In this case, condition (3.11) should be replaced by (2.32) and (2.33) or by (2.32) and (2.43) and the existence of a bound set is derived, respectively, from Theorems 2.8 and 2.11. Notice, in particular, that when using (2.32) and (2.43), we need to replace assumption (5) by

$$
|G(t, x(t), q(t), \lambda)| \leq c
$$

for all $t \in[a, b]$ and $(q, \lambda, x) \in \Gamma_{T}$, where $c$ is a positive constant. Alternatively, we can take condition (3.13) but with $d(t) \equiv d$, that is constant.

\section{Bounded solutions}

In this section, we combine Theorem 3.2 with a classical sequential approach in order to obtain the existence of a solution for inclusion (1.1) which is bounded on all the real line. Throughout this section, we will assume the multifunction $F$ 
to satisfy the growth assumption

$$
|F(t, x)| \leq c(t), \quad \forall(t, x) \in \mathbb{R} \times B_{0}^{R}
$$

where $c \in L_{\text {loc }}^{1}(\mathbb{R})$.

For this aim, we extend to multifunctions the known convergence result for sequences of uniformly bounded solutions of differential equations due to Krasnosel'skii [34]. For the sake of completeness, we also provide its proof which is based on the combination of classical techniques (see, e.g., $[13,19])$.

Lemma 4.1. Let $F: \mathbb{R} \times \mathbb{R}^{N} \rightsquigarrow \mathbb{R}^{N}$ be a Carathéodory set-valued map with nonempty, compact, and convex values satisfying (4.1) and let $\left\{x_{n}\right\}_{n}$ be a sequence of absolutely continuous functions such that

(1) for every $n \in \mathbb{N}, x_{n}$ is a solution of (1.1) defined in $[-n, n]$;

(2) $\sup \left\{\left|x_{n}(t)\right|: n \in \mathbb{N}, t \in[-n, n]\right\}=R<+\infty$.

Then (1.1) has a bounded solution on $\mathbb{R}$ whose values are contained in $B_{0}^{R}$.

Proof. For every $n \in \mathbb{N}$, denote by $\tilde{x}_{n}$ a suitable continuous extension of $x_{n}$ to $\mathbb{R}$ which is constant outside $[-n, n]$. Inequality (4.1) and (1), (2) imply that

$$
\left|\tilde{x}_{n}^{\prime}(t)\right| \leq c(t)
$$

for all $n \in \mathbb{N}$ and $t \in \mathbb{R}$.

Hence, since $c \in L_{\text {loc }}^{1}(\mathbb{R}),\left\{\tilde{x}_{n}^{\prime}\right\}_{n}$ is bounded in $L_{\text {loc }}^{1}(\mathbb{R})$. Thus, the BanachAlaoglu-Bourbaki's theorem (see, e.g., $[13,30]$ ) yields the existence of a subsequence, again denoted as the sequence, which locally weakly converges into $L_{\text {loc }}^{1}(\mathbb{R})$.

Moreover, since $c \in L_{\text {loc }}^{1}(\mathbb{R})$, and each $\tilde{x}_{n}$ is locally absolutely continuous, for every $\epsilon>0$, there exists $\delta>0$ such that, for all $t$ and $t^{\prime}$ with $\left|t-t^{\prime}\right| \leq \delta$,

$$
\left|\tilde{x}_{n}\left(t^{\prime}\right)-\tilde{x}_{n}(t)\right|=\left|\int_{t}^{t^{\prime}} \tilde{x}_{n}^{\prime}(s) d s\right| \leq \int_{t}^{t^{\prime}} c(s) d s \leq \epsilon .
$$

It follows that $\left\{\tilde{x}_{n}\right\}_{n}$ is locally equicontinuous in $C(\mathbb{R})$.

Then, because of (2), by the Ascoli-Arzelà lemma, we get the existence of a locally absolutely continuous function $x: \mathbb{R} \rightarrow B_{0}^{R}$ such that $\left\{\tilde{x}_{n}\right\}_{n}$ has a subsequence, again denoted as the sequence, which uniformly converges to $x$ on the compact subsets of $\mathbb{R}$.

Therefore, since, for every $t \in \mathbb{R}$,

$$
\begin{gathered}
\tilde{x}_{n}(t)-\tilde{x}_{n}(0)=\int_{0}^{t} \tilde{x}_{n}^{\prime}(s) d s \longrightarrow \int_{0}^{t} w(s) d s, \\
\tilde{x}_{n}(t)-\tilde{x}_{n}(0) \longrightarrow x(t)-x(0)=\int_{0}^{t} x^{\prime}(s) d s,
\end{gathered}
$$

it follows that $x^{\prime}=w$ a.e. in $\mathbb{R}$, that is, $\left\{\tilde{x}_{n}^{\prime}\right\}_{n} \rightarrow x^{\prime}$, locally weakly in $L_{\text {loc }}^{1}(\mathbb{R})$. 
By Mazur's convexity theorem (see, e.g., [30]), for each $n \in \mathbb{N}$, there exists a convex combination $z_{n}$ of $\left\{\tilde{x}_{m}^{\prime}\right\}_{m \geq n}$ such that $\left\{z_{n}\right\}_{n} \rightarrow x^{\prime}$ locally in $L_{\text {loc }}^{1}(\mathbb{R})$. Hence, passing to a subsequence, again denoted as the sequence, $\left\{z_{n}\right\}_{n} \rightarrow x^{\prime}$, locally a.e. in $\mathbb{R}$.

Recalling (1), consider now $M \subset \mathbb{R}$, with $\lambda(M)=0$, such that $\left\{z_{n}(t)\right\}_{n} \rightarrow x^{\prime}(t)$ locally, and $\tilde{x}_{n}^{\prime}(t) \in F\left(t, \tilde{x}_{n}(t)\right)$, for every $t \notin M$ and $n \geq|t|$.

Fix $t \notin M$. Given $\epsilon>0$, since $F$ is a Carathéodory map, it is then possible to find $\delta>0$ satisfying $F(t, y) \subset F(t, x(t))+B_{0}^{\epsilon}$, for all $y \in B_{x(t)}^{\delta}$.

Take now $\bar{n} \geq|t|$ such that, for every $n \geq \bar{n},\left|\tilde{x}_{n}(t)-x(t)\right| \leq \delta$, which is locally possible, because $\tilde{x}_{n}(t) \rightarrow x(t)$.

Notice that, for all $n \geq \bar{n}, \tilde{x}_{n}(t)=x_{n}(t)$ and this yields $\tilde{x}_{n}^{\prime}(t) \in F\left(t, \tilde{x}_{n}(t)\right) \subset$ $F(t, x(t))+B_{0}^{\epsilon}$. Consequently,

$$
\bigcup_{m \geq n} \tilde{x}_{m}^{\prime}(t) \in F(t, x(t))+B_{0}^{\epsilon} .
$$

Since $F$ is convex-valued, it implies that, for $n \geq \bar{n}$,

$$
\operatorname{conv}\left(\bigcup_{m \geq n} \tilde{x}_{m}^{\prime}(t)\right) \subset F(t, x(t))+B_{0}^{\epsilon},
$$

and so, $F$, being also compact-valued,

$$
\bigcap_{n \in \mathbb{N}} \overline{\operatorname{conv}\left(\bigcup_{m \geq n} \tilde{x}_{m}^{\prime}(t)\right)} \subset F(t, x(t))+B_{0}^{\epsilon} .
$$

Thus, for the arbitrariness of $\epsilon$,

$$
\bigcap_{n \in \mathbb{N}} \overline{\operatorname{conv}\left(\bigcup_{m \geq n} \tilde{x}_{m}^{\prime}(t)\right)} \subset F(t, x(t)) .
$$

Finally, since locally $\left\{z_{n}(t)\right\}_{n} \rightarrow x^{\prime}(t)$ and, by definition, $z_{m}(t) \in \operatorname{conv}\left(\cup_{p \geq n} \tilde{x}_{p}^{\prime}(t)\right)$, for every $m \geq n$, we obtain that, for every $n \in \mathbb{N}$,

$$
x^{\prime}(t) \in \overline{\operatorname{conv}\left(\bigcup_{m \geq n} \tilde{x}_{m}^{\prime}(t)\right)} .
$$

Therefore,

$$
x^{\prime}(t) \in \bigcap_{n \in \mathbb{N}} \overline{\operatorname{conv}\left(\bigcup_{m \geq n} \tilde{x}_{m}^{\prime}(t)\right)} \subset F(t, x(t)),
$$

for every $t \notin M$, and the result is proven.

Now, we give sufficient conditions for the existence of a bounded solution of (1.1). 
Theorem 4.2. Consider inclusion (1.1). Assume that

(1) there exists a bounded, nonempty, and open set $K \subset \mathbb{R}^{N}$ such that $K$ is symmetric with respect to the origin and $\bar{K}$ is a retract of $\mathbb{R}^{N}$; for every $\xi \in \partial K$, there exists a locally Lipschitzian function $V_{\xi}: \mathbb{R}^{N} \rightarrow \mathbb{R}$ satisfying

$$
\begin{aligned}
& V_{\xi}(\xi)=0, \\
& V_{\xi}(x) \leq 0, \quad \forall x \in \bar{K} \text { in a neighbourhood of } \xi
\end{aligned}
$$

(2) there exists a Carathéodory multifunction $G: \mathbb{R} \times \mathbb{R}^{N} \times \mathbb{R}^{N} \times[0,1] \rightsquigarrow \mathbb{R}^{N}$ with nonempty, compact, and convex values such that

$$
G(t, p, p, 1) \subset F(t, p), \quad \forall(t, p) \in \mathbb{R} \times \mathbb{R}^{N} ;
$$

(3) for every $t \in \mathbb{R}, q \in \bar{K}$, and $\lambda \in[0,1], G(t, \cdot, q, \lambda)$ is Lipschitzian with a sufficiently small Lipschitz constant;

(4) there exists a locally integrable function $c: \mathbb{R} \rightarrow \mathbb{R}$ satisfying

$$
|G(t, x, y, \lambda)| \leq c(t)(1+|x|), \quad \forall(t, x, y, \lambda) \in \mathbb{R} \times \mathbb{R}^{N} \times \bar{K} \times[0,1],
$$

where $c \in L_{\mathrm{loc}}^{1}(\mathbb{R})$;

(5) for every $n \in \mathbb{N}, T_{n}(Q \times\{0\}) \subset Q$, where $Q=\left\{q \in C\left(\mathbb{R}, \mathbb{R}^{N}\right): q(t) \in \bar{K}\right.$, for all $t\}, T_{n}$ denotes the map which assigns to all $(q, \lambda) \in Q \times[0,1]$ the set of solutions of

$$
\begin{gathered}
x^{\prime} \in G(t, x, q(t), \lambda), \quad \text { for a.a. } t \in[-n, n], \\
x(-n)=-x(n),
\end{gathered}
$$

and $\partial Q$ is fixed point free, that is, $\left\{q \in Q: q \in T_{n}(q, 0)\right\} \cap \partial Q=\varnothing$;

(6) for every $t \in \mathbb{R}, \xi \in \partial K, x \in \bar{K}$ in a neighbourhood of $\xi$, and $(q, \lambda) \in Q \times$ ]0,1], one has

$$
\liminf _{h \rightarrow 0^{-}} \frac{V_{\xi}(x+h w)-V_{\xi}(x)}{h}<0, \quad \forall w \in G(t, x, q(t), \lambda) .
$$

Then (1.1) admits a bounded solution.

Proof. Given $n \in \mathbb{N}$, consider the following antiperiodic boundary value problem on $[-n, n]$ :

$$
\begin{gathered}
x^{\prime} \in F(t, x), \quad \text { for a.a. } t \in[-n, n], \\
x(-n)=-x(n),
\end{gathered}
$$

that is, problem (3.1) with $M=-I$ and $A \equiv 0$, whose associated homogeneous problem has only the trivial solution.

Since $\overline{\mathscr{K}}=[-n, n] \times \bar{K}$ is a retract of $[-n, n] \times \mathbb{R}^{N}$ by condition $(1)$, and $\partial K=$ $[-n, n] \times \partial K$ is closed, then $K$ is an autonomous bound set for (4.16), for each 
$n \in \mathbb{N}$, because (2.3) is equivalent to the symmetry of $\partial K$ with respect to the origin.

Therefore, Theorem 3.2, whose condition (5) follows immediately from the present assumption (4) by means of the arguments in the proof of [14, Theorem 4], implies the existence of a solution of (4.16), that is, in particular, of an absolutely continuous function $x_{n}:[-n, n] \rightarrow \mathbb{R}$, satisfying (1.1).

Moreover, $x_{n}(t) \in \bar{K}$, for each $n \in \mathbb{N}$ and $t \in[-n, n]$, and the conclusion follows by Lemma 4.1, because $K$ is bounded.

Remark 4.3. In line with Remark 3.3, we leave to the reader the formulation of an existence result of bounded solutions of (1.1) using continuous bounding functions.

Remark 4.4. Similar results can be obtained sequentially when replacing the antiperiodic boundary value problems by a one-parameter family of different Floquet problems.

Remark 4.5. In the globally upper semicontinuous case, condition (6) can be replaced by those recalled in Remark 2.5 which are only localized in one point.

Example 4.6. Consider the differential inclusion

$$
x^{\prime} \in F_{1}(t, x)+F_{2}(t, x)
$$

where $F_{1}, F_{2}: \mathbb{R} \times \mathbb{R}^{N} \rightsquigarrow \mathbb{R}^{N}$ are Carathéodory multifunctions with nonempty, convex, and compact values such that there exist $c_{1}, c_{2} \in L_{\mathrm{loc}}^{1}(\mathbb{R})$, satisfying

$$
\begin{aligned}
& \left|F_{1}(t, 0)\right| \leq c_{1}(t), \quad \forall t \in \mathbb{R}, \\
& \left|F_{2}(t, x)\right| \leq c_{2}(t), \quad \forall(t, x) \in \mathbb{R} \times \bar{K}
\end{aligned}
$$

( $K$ is defined below) and $F_{1}(t, \cdot)$ is Lipschitzian, with a sufficiently small Lipschitz constant $L$, for all $t \in \mathbb{R}\left(\Rightarrow\left|F_{1}(t, x)\right| N+1 \leq L|x|+\left|F_{1}(t, 0)\right| \leq L|x|+c_{1}(t) \leq\right.$ $\left.\left(L+c_{1}(t)\right)(1+|x|) \forall(t, x) \in \mathbb{R}\right)$.

Assume, furthermore, the existence of positive constants $\epsilon$ and $R_{j}, j=1, \ldots$, $N$, such that

$$
\begin{gathered}
\left.K=\Pi_{j=1}^{N}\right]-R_{j}, R_{j}\left[, \quad \partial K_{j}=\left\{\xi \in \partial K: \xi_{j}= \pm R_{j}\right\},\right. \\
Q=\{q \in C(\mathbb{R}): q(t) \in \bar{K} \forall t \in \mathbb{R}\},
\end{gathered}
$$

and take, for all $j=1, \ldots, N, \xi \in \partial K_{j}, x \in \bar{K} \cap B_{\xi}^{\epsilon}, t \in \mathbb{R}, q \in Q$, and $w \in F_{1}(t, x)+$ $F_{2}(t, q(t))$, satisfying $\left(\operatorname{sign} \xi_{j} \cdot w_{j}\right) \neq 0$. Consider the family of multifunctions defined as $G(t, x, q, \lambda)=\lambda\left(F_{1}(t, x)+F_{2}(t, q)\right)$, which, recalling the growth conditions imposed on $F_{1}, F_{2}$, and the boundedness of $K$, satisfies assumptions (2), (3), and (4) of Theorem 4.2, with $c(t)=L+c_{1}(t)+c_{2}(t)$. Moreover, assumption 
(5) is trivially satisfied because the only solution of

$$
\begin{gathered}
x^{\prime}=0, \\
x(-n)=-x(n)
\end{gathered}
$$

is $x \equiv 0 \in \operatorname{int} Q$.

Finally, recalling Example 2.6, $K$ is a bound set for each problem

$$
\begin{gathered}
x^{\prime} \in G(t, x, q(t), \lambda), \quad \text { for a.a. } t \in[-n, n], \\
x(-n)=-x(n),
\end{gathered}
$$

with $\lambda \in] 0,1]$, and we get the existence of a bounded solution for (4.17) by Theorem 4.2.

\section{Concluding remarks}

The existence of bounded solutions of a rather general class of differential inclusions was investigated in Euclidean spaces.

The crucial step in our investigation consists in solving a one-parameter family of Floquet problems by means of a bound sets technique.

We took into account both locally Lipschitzian as well as continuous bounding functions. We also considered separately the cases of globally upper semicontinuous as well as Carathéodory right-hand sides.

On each level of regularity, the required conditions occurred to be specific. Hence, jointly with the previous paper [10] by the same authors, we have developed a rather complete theory of bound sets for inclusions.

In [6], we have recently made an extension of Proposition 3.1 in Banach spaces. On the other hand, it is known (see, e.g., [11, pages 120-125] and [16]) that typical bounding functions can be constructed neither in general Banach nor in Hilbert spaces, but only in $L^{2}$-spaces. So, a natural question arises about a generalization of our results into $L^{2}$-spaces. We will treat this elsewhere.

Another interesting class of problems might be related to retarded functional differential inclusions. The nontrivial examples of application of our methods to concrete differential equations and inclusions deserve our future interest as well.

\section{Acknowledgments}

We are indebted to our colleagues Prof. C. Castaing (University of Montpellier), Prof. J. Mawhin (Catholic University, Louvian-la-Neuve), Dr. K. Pastor (Palacký University, Olomouc), and Prof. M. Väth (University of Würzburg) for fruitful discussions and helpful suggestions. J. Andres is supported by the Council of Czech Government (J14/98: 153100 011) and by the Grant 201-00-0768 of the Grant Agency of Czech Republic. L. Malaguti and V. Taddei are supported by GNAFA-CNR and MURST within the Project "Metodi e problemi in Analisi Reale." 


\section{References}

[1] S. Ahmad and A. Tineo, Almost periodic solutions of second order systems, Appl. Anal. 63 (1996), no. 3-4, 389-395.

[2] J. M. Alonso, J. Mawhin, and R. Ortega, Bounded solutions of second order semilinear evolution equations and applications to the telegraph equation, J. Math. Pures Appl. (9) 78 (1999), no. 1, 49-63.

[3] J. Andres, On the multivalued Poincaré operators, Topol. Methods Nonlinear Anal. 10 (1997), no. 1, 171-182.

[4] Almost-periodic and bounded solutions of Carathéodory differential inclusions, Differential Integral Equations 12 (1999), no. 6, 887-912.

[5] Using the integral manifolds to solvability of boundary value problems, Set Valued Mappings with Applications in Nonlinear Analysis (R. P. Agarwal and D. O’Regan, eds.), Ser. Math. Anal. Appl., vol. 4, Taylor and Francis, Singapore, 2002, pp. 27-38.

[6] J. Andres and R. Bader, Asymptotic boundary value problems in Banach spaces, J. Math. Anal. Appl. 274 (2002), no. 1, 437-457.

[7] J. Andres, G. Gabor, and L. Górniewicz, Boundary value problems on infinite intervals, Trans. Amer. Math. Soc. 351 (1999), no. 12, 4861-4903.

[8] - Acyclicity of solution sets to functional inclusions, Nonlinear Anal., Ser. A: Theory Methods 49 (2002), no. 5, 671-688.

[9] J. Andres and B. Krajc, Bounded solutions in a given set of differential systems, J. Comput. Appl. Math. 113 (2000), no. 1-2, 73-82.

[10] J. Andres, L. Malaguti, and V. Taddei, Floquet boundary value problems for differential inclusions: a bound sets approach, Z. Anal. Anwendungen 20 (2001), no. 3, 709725.

[11] J. M. Appell, A. S. Kalitvin, and P. P. Zabrejko, Partial Integral Operators and IntegroDifferential Equations, Monographs and Textbooks in Pure and Applied Mathematics, vol. 230, Marcel Dekker, New York, 2000.

[12] J.-P. Aubin, Viability Theory, Systems \& Control: Foundations \& Applications, Birkhäuser, Boston, Massachusetts, 1991.

[13] J.-P. Aubin and A. Cellina, Differential Inclusions, Grundlehren der Mathematischen Wissenschaften, vol. 264, Springer-Verlag, Berlin, 1984.

[14] A. Augustynowicz, Z. Dzedzej, and B. D. Gelman, The solution set to BVP for some functional-differential inclusions, Set-Valued Anal. 6 (1998), no. 3, 257-263.

[15] A. Bacciotti, F. Ceragioli, and L. Mazzi, Differential inclusions and monotonicity conditions for nonsmooth Lyapunov functions, Set-Valued Anal. 8 (2000), no. 3, 299 309.

[16] E. A. Biberdorf, Stabilität und Beschränkheit bei Integro-Differentialgleichungen vom Barbashin-Typ, Diploma-thesis, University of Würzburg, 1995.

[17] C. Castaing and M. D. P. Monteiro Marques, $B V$ periodic solutions of an evolution problem associated with continuous moving convex sets, Set-Valued Anal. 3 (1995), no. 4, 381-399.

[18] _ Periodic solutions of evolution problem associated with moving convex sets, Discuss. Math. Differential Incl. 15 (1995), no. 2, 99-127.

[19] C. Castaing and M. Valadier, Convex Analysis and Measurable Multifunctions, Lecture Notes in Mathematics, vol. 580, Springer-Verlag, Berlin, 1977.

[20] F. H. Clarke, Yu. S. Ledyaev, and R. J. Stern, Asymptotic stability and smooth Lyapunov functions, J. Differential Equations 149 (1998), no. 1, 69-114. 
[21] F. H. Clarke, R. J. Stern, and P. R. Wolenski, Subgradient criteria for monotonicity, the Lipschitz condition, and convexity, Canad. J. Math. 45 (1993), no. 6, 1167-1183.

[22] E. N. Dancer, Boundary-value problems for ordinary differential equations on infinite intervals, Proc. London Math. Soc. (3) 30 (1975), 76-94.

[23] _ Boundary-value problems for ordinary differential equations of infinite intervals. II, Quart. J. Math. Oxford Ser. (2) 28 (1977), no. 109, 101-115.

[24] F. S. de Blasi, L. Górniewicz, and G. Pianigiani, Topological degree and periodic solutions of differential inclusions, Nonlinear Anal., Ser. A: Theory Methods 37 (1999), no. $2,217-243$.

[25] K. Deimling, Multivalued Differential Equations, de Gruyter Series in Nonlinear Analysis and Applications, vol. 1, Walter de Gruyter, Berlin, 1992.

[26] A. Fonda and F. Zanolin, Bounded solutions of nonlinear second order ordinary differential equations, Discrete Contin. Dynam. Systems 4 (1998), no. 1, 91-98.

[27] R. E. Gaines and J. L. Mawhin, Coincidence Degree, and Nonlinear Differential Equations, Lecture Notes in Mathematics, vol. 568, Springer-Verlag, Berlin, 1977.

[28] L. Górniewicz and S. Plaskacz, Periodic solutions of differential inclusions in $\mathbf{R}^{n}$, Boll. Un. Mat. Ital. A (7) 7 (1993), no. 3, 409-420.

[29] A. Granas, R. B. Guenther, J. W. Lee, and D. O'Regan, Boundary value problems on infinite intervals and semiconductor devices, J. Math. Anal. Appl. 116 (1986), no. 2, 335-348.

[30] S. Hu and N. S. Papageorgiou, Handbook of Multivalued Analysis. Vol. I. Theory, Mathematics and Its Applications, vol. 419, Kluwer Academic Publishers, Dordrecht, 1997.

[31] R. Kannan and C. K. Krueger, Advanced Analysis on the Real Line, Universitext, Springer-Verlag, New York, 1996.

[32] W. Karpińska, A note on bounded solutions of second order differential equations at resonance, Topol. Methods Nonlinear Anal. 14 (1999), no. 2, 371-384.

[33] A. M. Krasnosel'skii, M. A. Krasnosel'skii, J. Mawhin, and A. Pokrovskiü, Generalized guiding functions in a problem on high frequency forced oscillations, Nonlinear Anal. 22 (1994), no. 11, 1357-1371.

[34] M. A. Krasnosel'ski1, The Operator of Translation Along the Trajectories of Differential Equations, Translations of Mathematical Monographs, vol. 19, American Mathematical Society, Rhode Island, 1968.

[35] M. A. Krasnosel'skiı̆, J. Mawhin, and A. Pokrovskiŭ, New theorems on forced periodic oscillations and bounded solutions, Soviet Phys. Dokl. 36 (1991), no. 11, 743-745.

[36] M. Lewicka, Locally Lipschitzian guiding function method for ODEs, Nonlinear Anal. 33 (1998), no. 7, 747-758.

[37] J. W. Macki, P. Nistri, and P. Zecca, The existence of periodic solutions to nonautonomous differential inclusions, Proc. Amer. Math. Soc. 104 (1988), no. 3, 840844.

[38] L. Malaguti, Bounded solutions for a class of second order nonlinear differential equations, Differential Equations Dynam. Systems 3 (1995), no. 2, 175-188.

[39] L. Malaguti and C. Marcelli, Existence of bounded trajectories via upper and lower solutions, Discrete Contin. Dynam. Systems 6 (2000), no. 3, 575-590.

[40] M. Marcus and V. J. Mizel, Absolute continuity on tracks and mappings of Sobolev spaces, Arch. Rational Mech. Anal. 45 (1972), 294-320.

[41] J. Mawhin, Bound sets and Floquet boundary value problems for nonlinear differential equations, Univ. Iagel. Acta Math. (1998), no. 36, 41-53. 
[42] J. Mawhin and J. R. Ward Jr., Bounded solutions of some second order nonlinear differential equations, J. London Math. Soc. (2) 58 (1998), no. 3, 733-747.

[43] - Guiding-like functions for periodic or bounded solutions of ordinary differential equations, Discrete Contin. Dynam. Systems 8 (2002), no. 1, 39-54.

[44] R. Ortega, A boundedness result of Landesman-Lazer type, Differential Integral Equations 8 (1995), no. 4, 729-734.

[45] R. Ortega and A. Tineo, Resonance and non-resonance in a problem of boundedness, Proc. Amer. Math. Soc. 124 (1996), no. 7, 2089-2096.

[46] S. Plaskacz, Periodic solutions of differential inclusions on compact subsets of $\mathbf{R}^{n}$, J. Math. Anal. Appl. 148 (1990), no. 1, 202-212.

[47] E. Roxin, Stability in general control systems, J. Differential Equations 1 (1965), 115150.

[48] V. Taddei, Bound sets for Floquet boundary value problems: the nonsmooth case, Discrete Contin. Dynam. Systems 6 (2000), no. 2, 459-473.

[49] B. S. Thompson, Real Functions, Lecture Notes in Mathematics, vol. 1170, SpringerVerlag, Berlin, 1985.

[50] F. Zanolin, Bound sets, periodic solutions and flow-invariance for ordinary differential equations in $\mathbf{R}^{n}$ : some remarks, Rend. Istit. Mat. Univ. Trieste 19 (1987), no. 1, 76-92.

Jan Andres: Department of Mathematical Analysis, Faculty of Science, Palacký University, Tomkova 40, 77900 Olomouc, Czech Republic

E-mail address: andres@risc.upol.cz

Luisa Malaguti: Department of Engineering Sciences and Methods, University of Modena and Reggio Emilia, Via Fogliani 1, 42100 Reggio Emilia, Italy

E-mail address: malaguti.luisa@unimore.it

Valentina Taddei: Department of Mathematics and Informatics, University of Udine, Via delle Scienze 206, 33100 Udine, Italy

E-mail address: taddei.valentina@unimore.it 


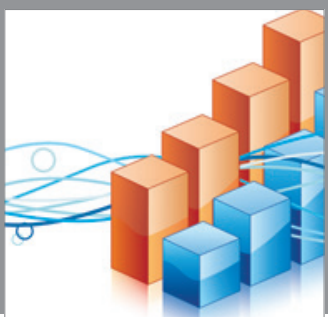

Advances in

Operations Research

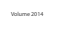

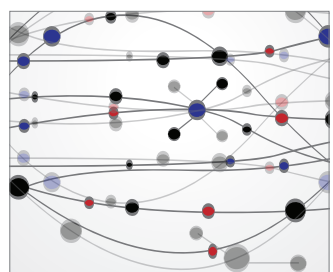

\section{The Scientific} World Journal
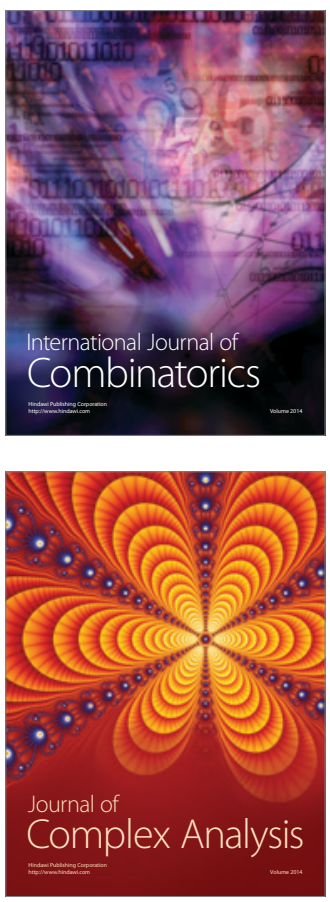

International Journal of

Mathematics and

Mathematical

Sciences
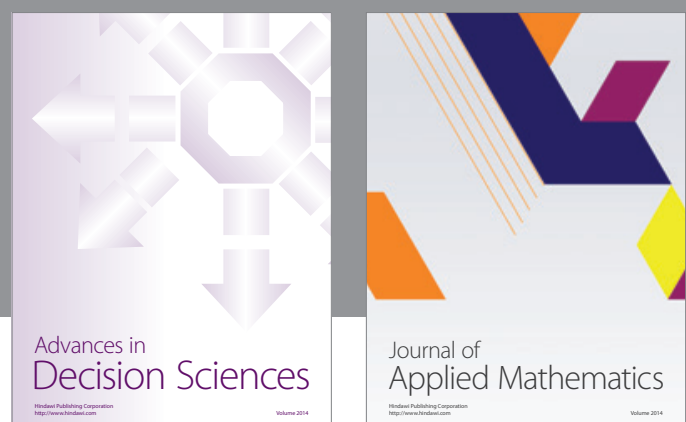

Journal of

Applied Mathematics
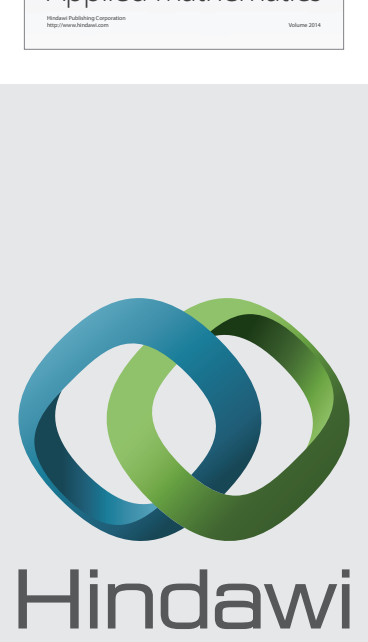

Submit your manuscripts at http://www.hindawi.com
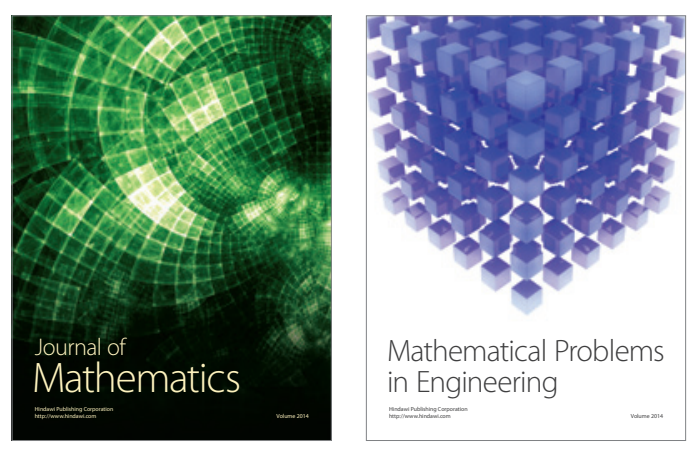

Mathematical Problems in Engineering
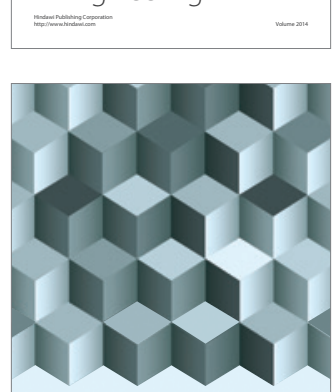

Journal of

Function Spaces
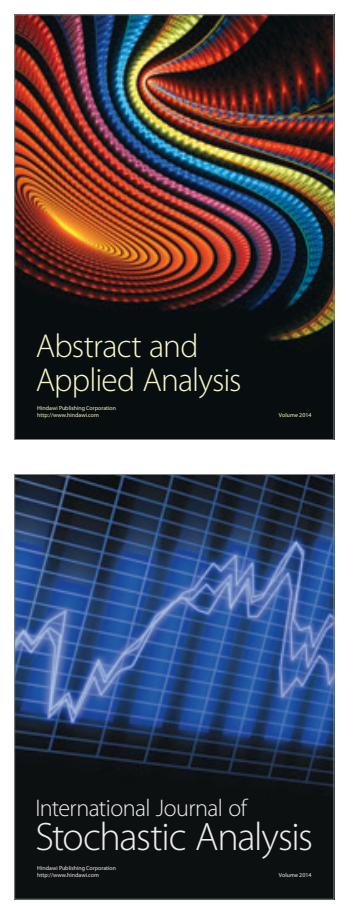

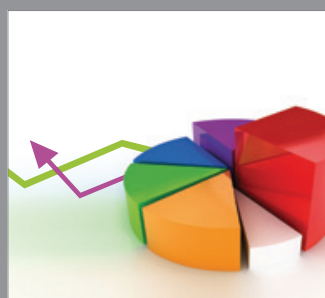

ournal of

Probability and Statistics

Promensencen
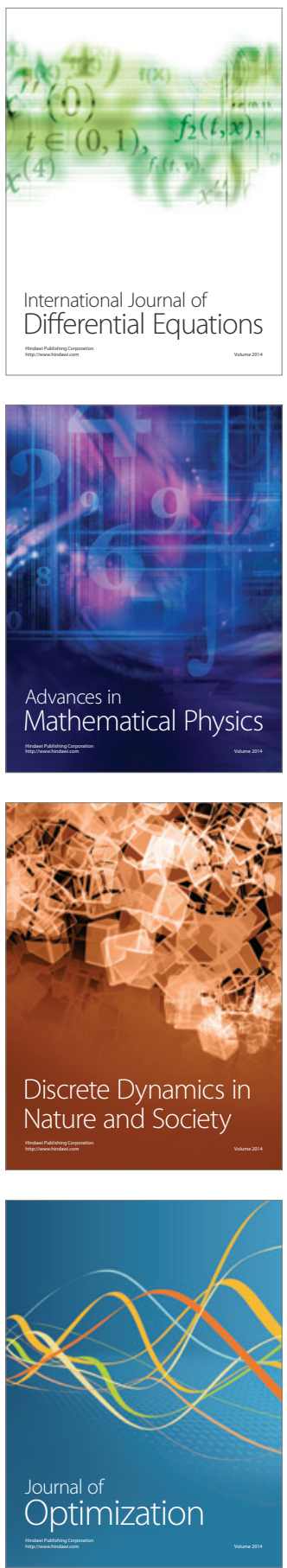